\title{
Astragalus polysaccharide induces the apoptosis of human hepatocellular carcinoma cells by decreasing the expression of Notch1
}

\author{
WEN-HAI HUANG*, WEI-RONG LIAO* and RONG-XUN SUN \\ Department of General Surgery, Jinshan Hospital Affiliated to Fudan University, Shanghai 201508, P.R. China
}

Received March 24, 2015; Accepted April 11, 2016

DOI: $10.3892 /$ ijmm.2016.2632

\begin{abstract}
Hepatocellular carcinoma (HCC) is the third most frequent cause of cancer death worldwide. Astragalus polysaccharide (APS), the primary active component extracted from a traditional Chinese medicinal herb Astragalus membranaceus, has been proved to exert a marked inhibitory effect on a number of types of human solid tumors. In the present study, we aimed to examine the effects of APS on the survival of the $\mathrm{HCC}$ cell line $\mathrm{H} 22$ and to elucidate the underlying regulatory mechanisms responsible for these effects. Our results revealed that the mRNA and protein expression of Notch1 was significantly upregulated in the HCC tissues compared with that in the normal tissues. APS decreased cell viability and induced the apoptosis of HCC cells in a concentration-dependent manner, which were evaluated using a cell counting kit-8 (CCK-8) assay and flow cytometric analysis, respectively. Furthermore, APS regulated the expression of apoptosis-related genes (Bcl-2 and BAX) and proteases (caspase-3 and -8). Mechanically, Notch1 expression was found to be suppressed in HCC cells, and further analysis indicated that Notch1 knockdown by siRNA significantly reduced cell viability, suppressed the metastatic capacity and enhanced the apoptosis of HCC cells. Taken together, these findings suggest that Notch1 may be a potential therapeutic target for the treatment of HCC.
\end{abstract}

\section{Introduction}

Hepatocellular carcinoma (HCC) is the most common primary hepatic malignancy, which is associated with increasing morbidity worldwide over the last decade (1). HCC is the third most common cause of cancer death in the world, and it accounts for $90 \%$ of primary liver cancers (2-5). Despite great

Correspondence to: Dr Rong-Xun Sun, Department of General Surgery, Jinshan Hospital Affiliated to Fudan University, 1508 Longhang Road, Shanghai 201508, P.R. China

E-mail: alex_sun78@163.com

${ }^{*}$ Contributed equally

Key words: Astragalus polysaccharide, apoptosis, hepatocellular carcinoma, Notch1 efforts to develop treatments for HCC, which have led to the implementation of several relatively effective methods, the cure rate and survival of patients with liver cancer are still not optimistic (6-9). At present, liver resection and transplantation remain the principal treatments for patients with early-stage HCC (10). However, tumor recurrence after liver resection and a lack of appropriate donor organs are still the major problems affecting patient survival (11-13). Thus, in-depth investigations of the molecular mechanisms underlying the development of HCC are urgently required, which may lead to novel therapies for HCC.

Astragalus polysaccharide (APS), the primary active constituent extracted from a traditional Chinese medicinal herb Astragalus membranaceus, has been shown to exhibit diverse pharmacological and biological effects, including antioxidant, immune-enhancing and antiviral effects as well as resistance to immunosuppression (14-20). Several studies have demonstrated the antiviral effects (specifically anti-duck hepatitis A virus activity and anti-infectious bursal disease virus activity) of APS and its sulfate (sAPS) in vitro $(21,22)$. In the tumor microenvironment of human HCC, it has been demonstrated that APS is capable of restoring the cytokine balance and suppressing the expression of FOXp3 mRNA, to inhibit the immunosuppressive effects of Treg cells (23). Furthermore, APS exerts a marked inhibitory effect on a number of types of solid tumors (24-26). A previous study suggested that Astragalus injection can suppress apoptosis of mesothelial cells, which has the ability to prevent cancer invasion, and thus revealed that Astragalus may be used in treatment of gastric cancer (25). Astragalus saponin extract induced growth inhibition and apoptosis in human colon cancer cells and a tumor xenograft model in nude mice (27). APS exerted a synergistic antitumor effect with adriamycin in $\mathrm{H} 22$ tumor-bearing mice by promoting the expression of interleukin (IL)-1 $\alpha$, IL-2, IL- 6 and tumor necrosis factor (TNF)- $\alpha$, and by suppressing the expression of IL-10, MDR1 mRNA and P-glycoprotein (P-GP) (28). Taken together, these studies show that despite evidence of APS exerting inhibitory effects on human solid tumors, and the use of APS as an adjuvant treatment in combination with other anticancer drugs in order to reduce side-effects and increase sensitivity, its effect on the progression of HCC as well as the underlying regulatory mechanism remain unclear.

Recent findings have indicated that the ectopic expression of Notch1 was associated with increased metastatic capacity, 
improved survival times and vasculogenic mimicry in HCC cell lines $(29,30)$. Notch proteins (Notch 1-4) are transmembrane receptors, which have an extracellular domain for binding to specific ligands and an intracellular domain involved in transcriptional regulation (31). Previous research has demonstrated that the Notch signaling pathway plays a number of important roles in cancer development (32-35). Notch1 signaling has been demonstrated to regulate cell proliferation, apoptosis and differentiation in lung carcinoma (34). The downregulation of Notch1 inhibited cell growth and induced apoptosis in A2780 ovarian cancer cells (35). A chemically sulfated polysaccharide derived from Grifola frondosa induced HepG2 cell apoptosis through the Notch1/nuclear factor (NF)- $\mathrm{kB} / \mathrm{p} 65$-mediated caspase pathway (36). A recent study indicated that Notch1 is a potential therapeutic target for APS-induced apoptosis in non-small cell lung carcinoma cell lines (37). Based on these findings, we hypothesized that APS may be involved in the regulation of tumor growth and metastasis through Notch1 signaling.

In the present study, we aimed to examine the effects of APS on the survival of an HCC cell line (H22) as well as the underlying regulatory mechanism responsible for these effects. Our results revealed that APS decreased cell viability and induced cell apoptosis in HCC cells by decreasing the expression of Notch1. Notch1 may be a potential therapeutic target for the treatment of HCC.

\section{Materials and methods}

Samples and cell culture. Human HCC samples were collected from patients who underwent surgery at Jinshan Hospital Affiliated to Fudan University (Shanghai, China). All the patients provided written informed consent. Dissected samples were frozen immediately after surgery and stored at $-80^{\circ} \mathrm{C}$ until use. All procedures involved clinical specimens were approved by the Ethics Committee of Jinshan Hospital Affiliated to Fudan University. The mouse HCC cell line (H22) was obtained from the Shanghai Cell Bank (Chinese Academy of Sciences) and cultured in RPMI-1640 medium (Invitrogen, Carlsbad, CA, USA) supplemented with $10 \%$ fetal bovine serum (FBS; Invitrogen), $100 \mu \mathrm{g} / \mathrm{ml}$ streptomycin and $100 \mathrm{U} / \mathrm{ml}$ penicillin solutions (both from Beyotime Institute of Biotechnology, Haimen, China). The cells were incubated at $37^{\circ} \mathrm{C}$ in humidified air with $5 \% \mathrm{CO}_{2}$. The medium buffer was replaced daily.

Reagents. APS was purchased from Hongsheng Biotech Co. (Xi'an, China) and was diluted in $\mathrm{H}_{2} \mathrm{O}$ immediately prior to administration.

Assays of cell viability. The cells were plated at a density of $1 \times 10^{4}$ cells per well in a 96-well plate. Following incubation, the cells were treated with or without APS. Cell viability was quantified using a cell counting kit-8 (CCK-8; Dojindo Laboratories, Kumamoto, Japan) for 3 consecutive days after infection. Each data point was obtained in triplicate.

Analysis of cell apoptosis. The rate of apoptosis was measured by Annexin-V FITC and PI staining, followed by flow cytometry (flow cytometer from Becton-Dickinson, Franklin Lakes, NJ, USA). Briefly, the cells were trypsinized and suspended in $500 \mu \mathrm{l}$ of binding buffer containing $5 \mu \mathrm{l}$ Annexin V FITC and 5 $\mu \mathrm{l}$ PI (Sigma-Aldrich Chemie Gmbh, Munich, Germany). Following incubation in the dark for $1 \mathrm{~h}$, the cells were subjected to flow cytometry and the rate of cell apoptosis was determined.

Reverse transcription-quantitative polymerase chain reaction $(R T-q P C R)$. Total RNA was extracted from the cells using TRIzol reagent (Invitrogen) according to the manufacturer's instructions. Reverse transcription was performed using a SuperScript III Reverse Transcriptase kit (Invitrogen) in accordance with the manufacturer's instructions. Quantitative (real-time) PCR (qPCR) was performed using an Applied Biosystems 7300 Sequence Detection system (Applied Biosystems, Foster City, CA, USA). The $20 \mu \mathrm{l}$ PCR reaction included $1 \mu \mathrm{l}$ of cDNA, $10 \mu \mathrm{l}$ of $2 \mathrm{X}$ TaqMan gene expression master mix and $1 \mu \mathrm{l}$ of TaqMan gene expression assays reagent (Applied Biosystems). The reactions were incubated in 96-well optical plates at $95^{\circ} \mathrm{C}$ for $10 \mathrm{~min}$, followed by 40 cycles of $95^{\circ} \mathrm{C}$ for $15 \mathrm{sec}$ and $60^{\circ} \mathrm{C}$ for $1 \mathrm{~min}$. qPCR reactions were performed in triplicate. The expression levels of the relative genes were calculated using the $2^{-\Delta \Delta \mathrm{CT}}$ method. GAPDH served as an internal control. The primer sets used were as follows: Notch1 forward, 5'-GATGACCTGGGCAAGTC-3' and reverse, 5'-CCCTGTTGTTCTGCATATCT-3'; GAPDH forward, 5'-GCACCGTCAAGCTGAGAAC-3' and reverse, 5'-GAGC AGCGTCTTCAGAGACAG-3'; Bcl-2 forward, 5'-CTGAGT ACCTGAACCGGCATC-3' and reverse, 5'-TGGTGAAGAC GCCAGTGGA-3'; BAX forward, 5'-GTTTCATCCAGGA TCGAGCAG-3' and reverse, 5'-AGCTGAGCGAGTGTCT CCGGCG -3'. The PCR products were separated on a $1.2 \%$ agarose gel and identified after ethidium bromide staining (both from Tiangen Biotech (Beijing) Co., Ltd, Beijing, China).

Western blot analysis. Protein extraction and western blot analysis were performed as previously described (29). $\beta$-actin was used as the loading control protein. Primary antibodies against Notch1, Bcl-2, BAX, caspase-3, caspase-8, E-cadherin, matrix metallopeptidase 9 (MMP-9), cyclooxygenase-2 (COX-2) and $\beta$-actin were all purchased from Santa Cruz Biotechnology (Santa Cruz, CA, USA). All secondary antibodies were obtained from Univ-Bio Inc. (Shanghai, China).

Transfection of small interfering RNA (siRNA). Three different siRNAs targeting Notch1 (si-Notch1-1, si-Notch1-2 and si-Notch1-3) and a scramble siRNA (siScramble) were synthesized by Santa Cruz Biotechnology and transfected into the HCC cells using Lipofectamine 2000 (Invitrogen) as previously described by Zhou et al (29). The sequences of the siRNAs were as follows: si-Notch1-1 sense, 5'-GCUCCCU CAACUUCAAUGAUU-3' and antisense, $3^{\prime}$-UUCGAGGGAG UUGAAGUUACU-5'; si-Notch1-2 sense, 5'-GCCUGGACAA GAUCAAUGAUU-3' and antisense, 3'-UUCGGACCUGUUC UAGUUACU-5'; si-Notch1-3 sense, 5'-CAGGGAGCAUG UGUAACAUUU-3' and antisense, 3'-UUGUCCCUCGUA CACAUUGUA-5'.

Statistical analysis. Statistical analysis was conducted using SPSS software version 16.0 (SPSS, Inc., Chicago, IL, USA). All experiments were performed at least three times, and the data 
A

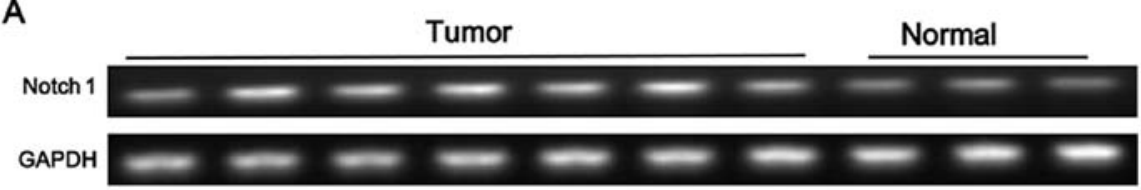

B

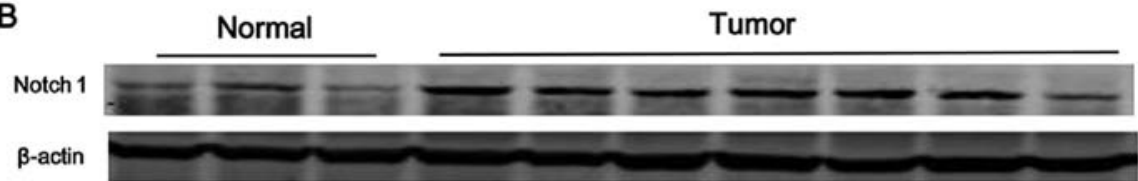

C

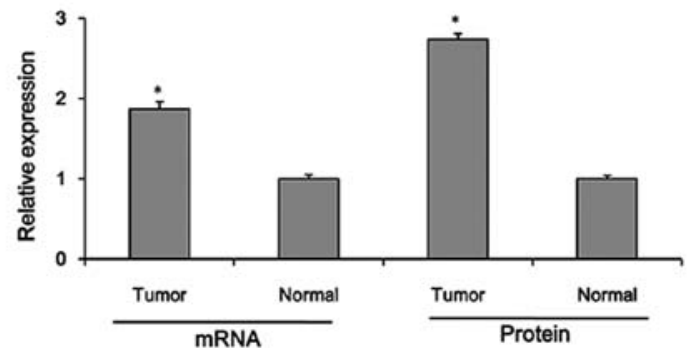

Figure 1. mRNA and protein levels of Notch1 in human hepatocellular carcinoma (HCC) tissues. (A) Notch1 mRNA expression in human HCC tissues (n=7) and normal liver tissues $(n=3)$. (B) Western blot analysis of Notch1 protein expression in human HCC tissues $(n=7)$ and normal liver tissues ( $n=3)$. (C) Relative expression of Notch1 in human HCC tissues ( $\mathrm{n}=7$ ) and normal liver tissues $\left(\mathrm{n}=3\right.$ ). Data are presented as the means $\pm \mathrm{SD},{ }^{*} \mathrm{P}<0.05$.

were summarized and are presented as the means $\pm \mathrm{SD}$. The t-test was used to compare two independent groups. Inter-group differences were analyzed by one-way ANOVA followed by Tukey's multiple comparison test as a post-test to compare the group means. $\mathrm{P}<0.05$ was considered to indicate a statistically significant difference.

\section{Results}

Notch1 is upregulated in HCC tissues. According to a study by Zhou et al (29), the results of immunohistochemical analysis revealed that the high expression of Notch1 in HCC tissues correlated with tumor size, tumor grade, metastasis and venous invasion. In the present study, we determined the protein and mRNA levels of Notch1 in seven HCC tissues and three normal liver tissues. As shown in Fig. 1, the protein and mRNA levels of Notch1 were significantly upregulated in the HCC tissues compared with those in normal tissues $(\mathrm{P}<0.05)$.

APS decreases cell viability and induces cell apoptosis in HCC cells. It was previously demonstrated that APS inhibited the growth and proliferation of Treg cells in the tumor microenvironment of HCC and exerted an antitumor effect $(23,28)$. To determine whether APS exerts an effect on the regulation of $\mathrm{HCC}$ cell growth, $\mathrm{H} 22$ cells were incubated with different concentrations of APS. Firstly, we examined the viability of the H22 cells. The results showed that APS treatment decreased cell survival in a concentration-dependent manner (Fig. 2A).

To determine whether APS induced cell death through an apoptotic mechanism, apoptosis was detected by Annexin V/PI double staining. As shown in Fig. 2B and C, the apoptotic rate was significantly increased in the $\mathrm{H} 22$ cells following APS treatment in a concentration-dependent manner. To further explore the effect of APS on apoptosis in the HCC cells, we measured the expression level of apoptosis-related genes (Bcl-2 and BAX). It was found that at concentrations $>0.1 \mathrm{mg} / \mathrm{ml}$, APS significantly inhibited the mRNA level of the apoptosis suppressor gene Bcl-2 (Fig. 3A). Similarly, the protein level of Bcl-2 was downregulated markedly following the incubation of the $\mathrm{H} 22$ cells with APS (Fig. $3 \mathrm{C}$ and D, P<0.01). In addition, the mRNA level of BAX, a pro-apoptotic gene, was markedly increased by APS in a concentration-dependent manner (Fig. 3B). Furthermore, the expression of the apoptosis-related proteases (caspase-3 and -8) was also affected by APS. Following APS treatment, the protein levels of caspase- 3 and -8 were significantly enhanced in the H22 cells (Fig. 3C and D). Taken together, these findings suggest that APS decreases HCC cell survival through an apoptotic mechanism.

APS inhibits Notchl expression in HCC. To further investigate the molecular mechanism underlying the inhibitory effect of APS on HCC cell survival, we evaluated the expression level of Notch1 in the APS-treated H22 cells. As shown in Fig. 4, APS treatment significantly decreased the mRNA and protein levels of Notch1 in the H22 cells in a concentration-dependent manner, which suggests that Notch1, is a potential target of APS.

Notch1 knockdown suppresses the survival and metastasis of HCC cells. After clarifying the association between APS and Notch1, we further intended to explore the effects of Notch1 on cell growth. Firstly, Notch1 was knocked down in the H22 cells. Three different siRNAs targeting Notch1 were transfected into the H22 cells. The Notch1 mRNA level was markedly downregulated and si-Notch1-3 exhibited the strongest inhibitory effect (Fig. 5A). Consequently, si-Notch1-3 was selected for use in subsequent experiments. As shown in Fig. 5B-D, Notch1 knockdown reduced cell viability and significantly enhanced apoptosis $(\mathrm{P}<0.05)$. The next experiment examined changes in 

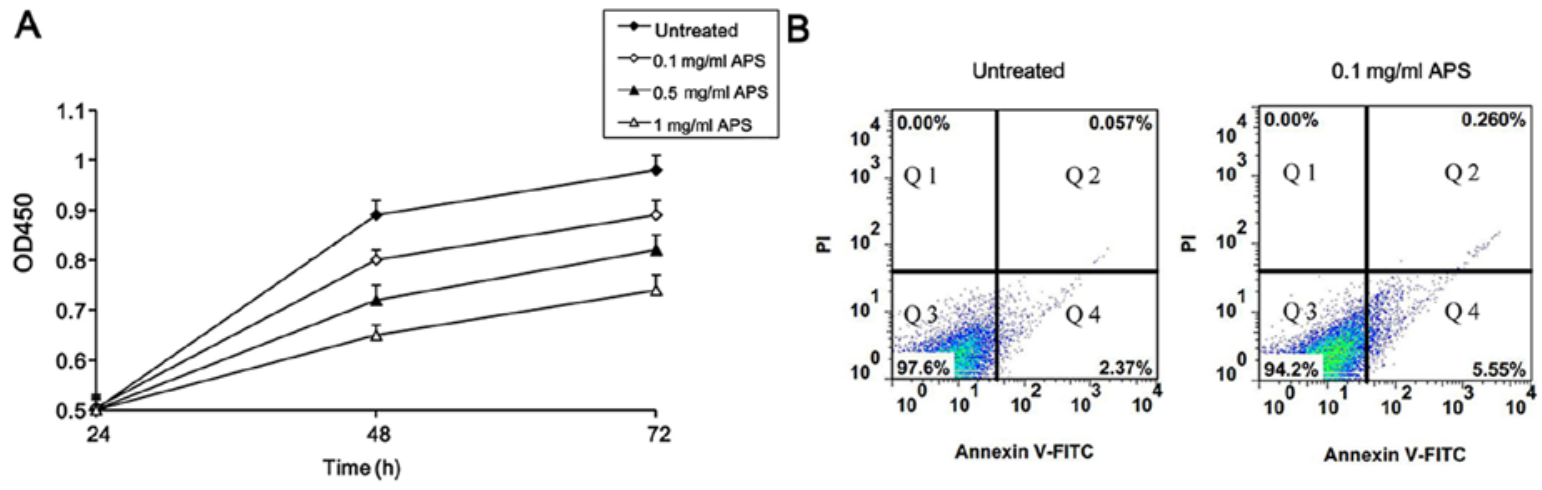

C
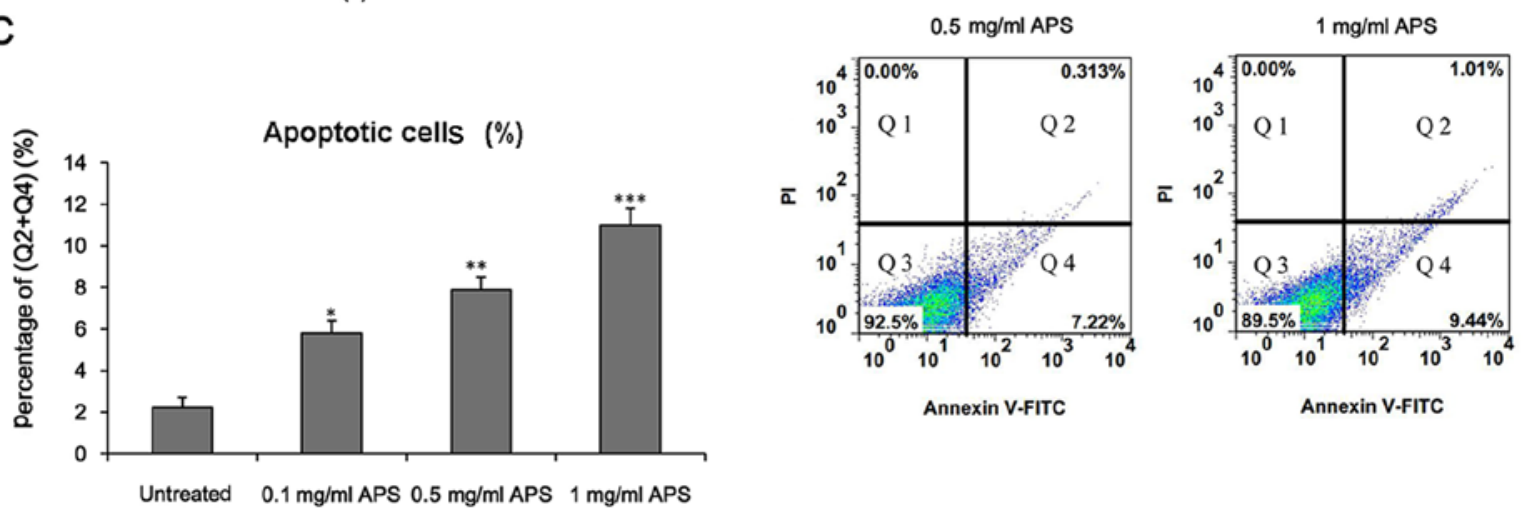

Figure 2. Astragalus polysaccharide (APS) inhibits cell survival and induces cell apoptosis in hepatocellular carcinoma (HCC) cells. (A) Cell viability of H22 cells following APS treatment (B) Flow cytometric analysis of apoptotic cells following APS treatment using Annexin V/PI double staining. (C) The percentage of apoptotic $\mathrm{H} 22$ cells following APS treatment. Data are presented as the means $\pm \mathrm{SD},{ }^{*} \mathrm{P}<0.05,{ }^{* *} \mathrm{P}<0.01,{ }^{* * * *} \mathrm{P}<0.001$. Q , quadrant.
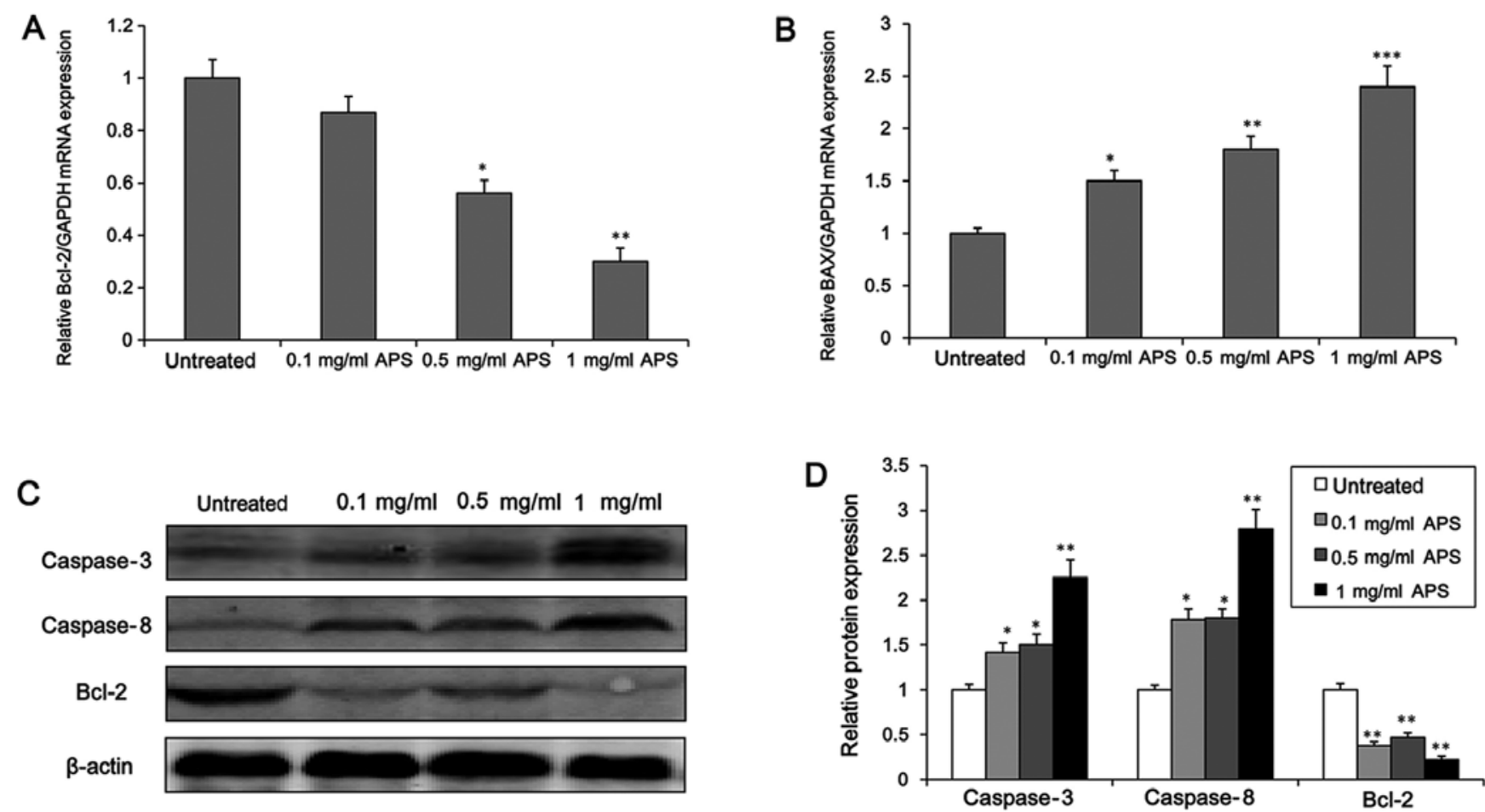

Figure 3. Astragalus polysaccharide (APS) regulates the expression of apoptosis-related genes and proteases in hepatocellular carcinoma (HCC) cells. (A) mRNA levels of Bcl-2 in $\mathrm{H} 22$ cells following APS treatment. (B) mRNA level of BAX in H22 cells following APS treatment. (C) Western blot analysis of the protein expression of caspase-3, caspase- 8 and Bcl-2 in H22 cells APS treatment. (D) The relative protein expression of caspase-3, caspase-8 and Bcl-2 in $\mathrm{H} 22$ cells following APS treatment. Data are presented as the means $\pm \mathrm{SD},{ }^{*} \mathrm{P}<0.05,{ }^{* *} \mathrm{P}<0.01,{ }^{* * * *} \mathrm{P}<0.001$.

the expression of apoptosis-related genes. Notch1 knockdown significantly suppressed the protein expression of the apoptosis suppressor gene, $\mathrm{Bcl}-2$, and enhanced the protein expression of the pro-apoptotic gene, BAX (Fig. 6A and B, P<0.05). Finally, 
A

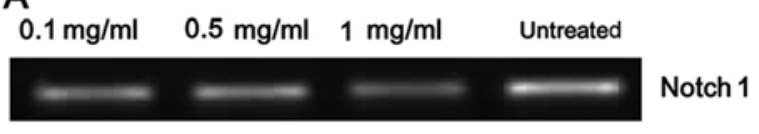

GAPDH

B

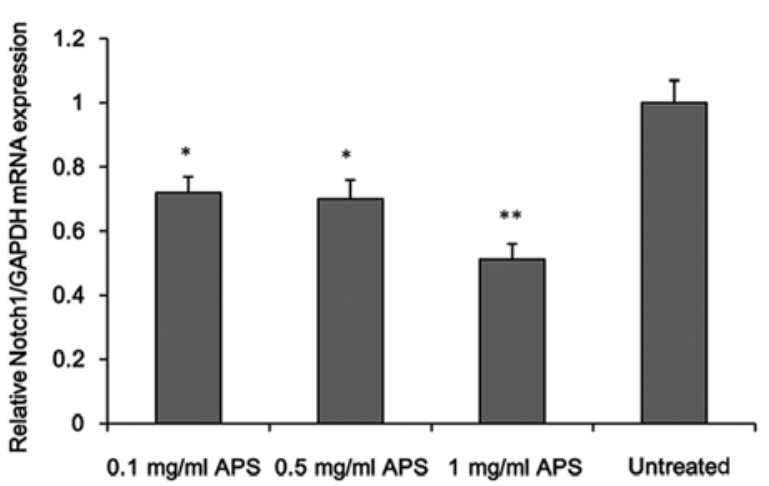

C

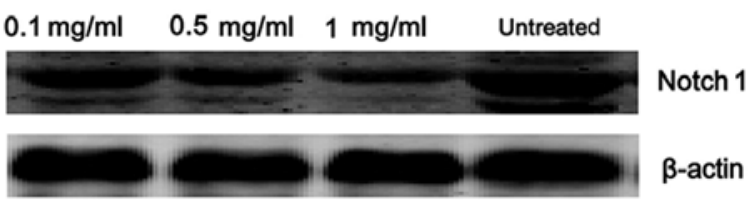

D

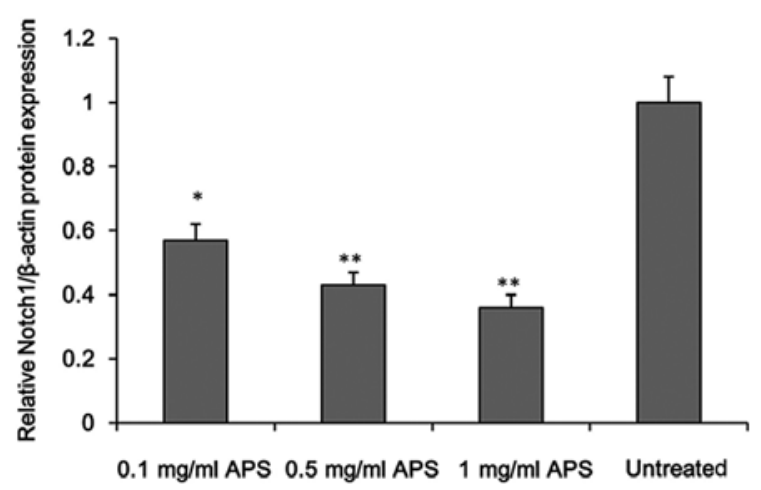

Figure 4. Astragalus polysaccharide (APS) inhibits the expression of Notch1 in hepatocellular carcinoma (HCC) cells. (A) Notch1 mRNA expression in the HCC cell line (H22) cells following APS treatment. (B) The relative mRNA expression of Notch1 in H22 cells following APS treatment. (C) Western blot analysis of Notch1 protein expression in H22 cells following APS treatment. (D) The relative protein expression of Notch1 in H22 cells following APS treatment. Data are the presented as the means $\pm \mathrm{SD},{ }^{*} \mathrm{P}<0.05,{ }^{* * *} \mathrm{P}<0.01$.

\section{A}

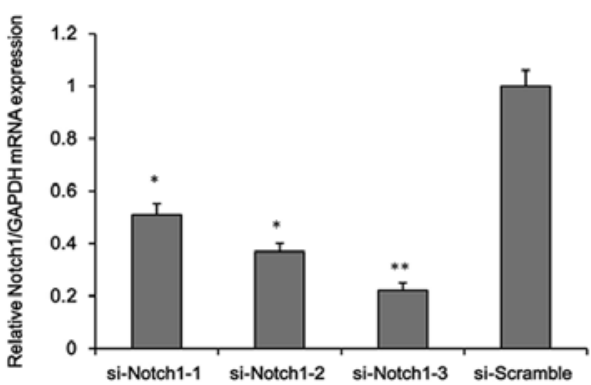

B

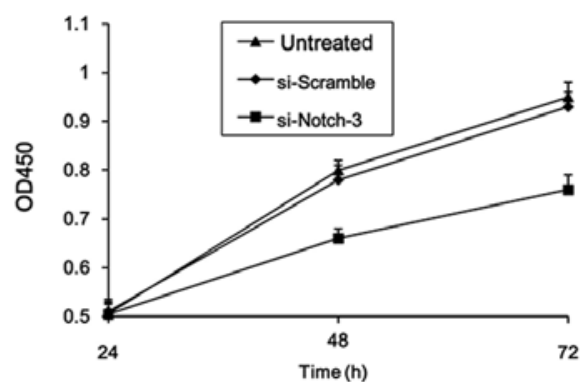

C

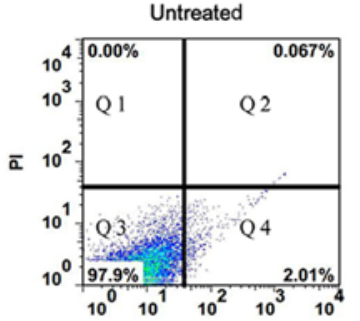

Annexin V-FITC

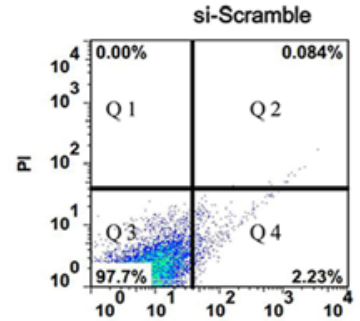

Annexin V-FITC

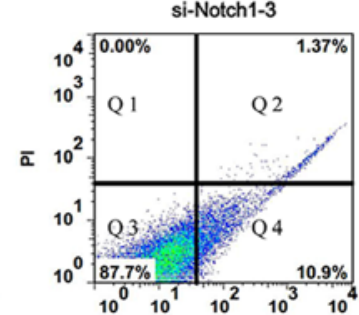

Annexin V-FITC

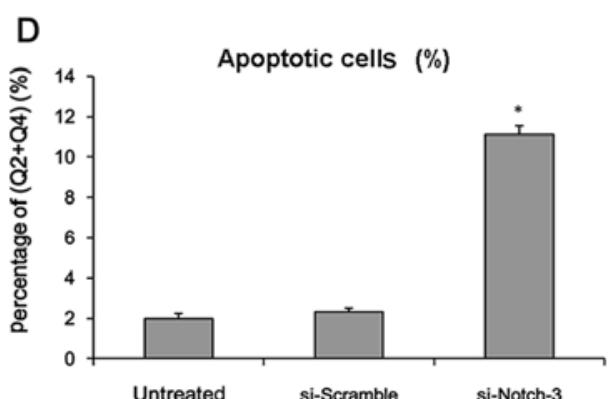

Figure 5. Notch1 knockdown decreases cell survival and induces cell apoptosis in hepatocellular carcinoma (HCC) cells. (A) The relative mRNA expression of Notch1 in the HCC cell line (H22) cells following siRNA transfection. (B) The cell viability of H22 cells following Notch1 knockdown. (C) Flow cytometric analysis of Annexin V/PI double staining following Notch1 knockdown. (D) The percentage of apoptotic H22 cells following Notch1 knockdown. Data are presented as the means \pm SD. ${ }^{*} \mathrm{P}<0.05,{ }^{* *} \mathrm{P}<0.01$. Q, quadrant.

the protein expression levels of metastasis-associated molecules including E-cadherin, MMP-9 and COX-2 were examined to determine whether Notch1 affects the metastasis of HCC cells. The results showed that the protein expression of E-cadherin was significantly increased by Notch1 knockdown, whereas the levels of MMP-9 and COX-2 were markedly reduced (Fig. 6C and $\mathrm{D}, \mathrm{P}<0.05$ ). These results suggest that Notch1 plays a role in the regulation of HCC cell survival and metastasis.

\section{Discussion}

In the present study, we evaluated changes in the expression of Notch1 in human HCC tissues compared with that in the normal tissues, and found that Notch1 was overexpressed in the HCC tissues. We further demonstrated that APS decreased the survival of HCC cells through an apoptotic mechanism. Furthermore, we showed that Notch1 knockdown decreased 
A

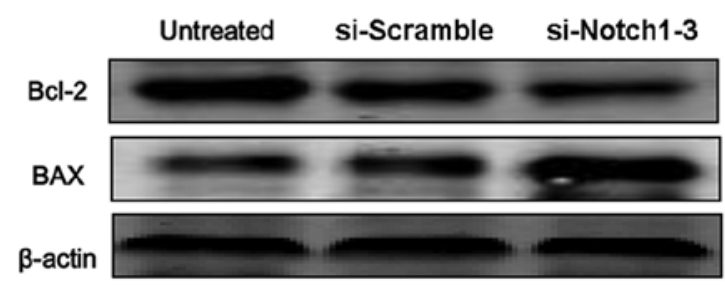

C

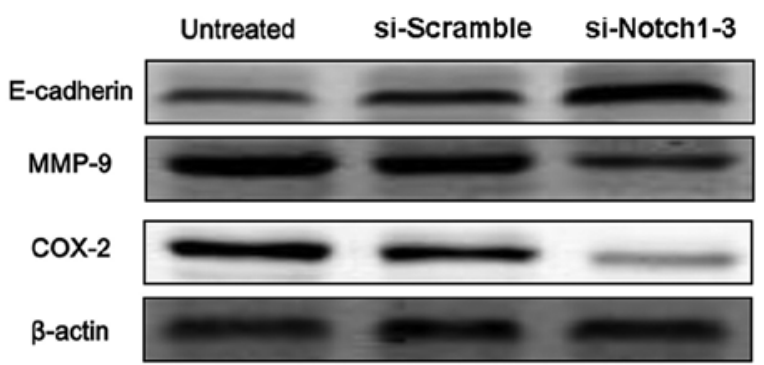

B

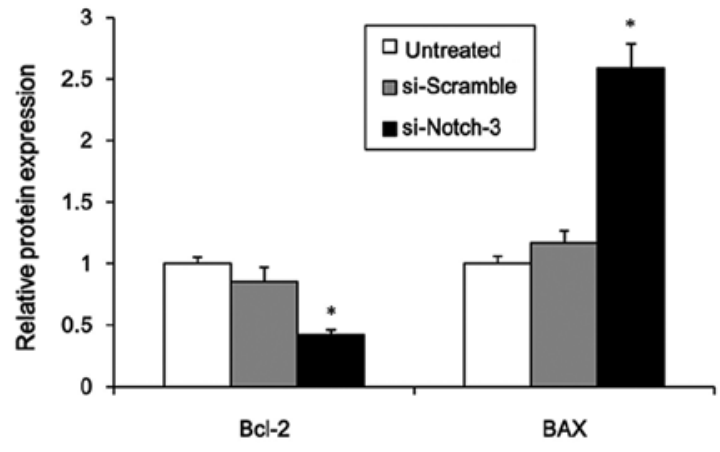

D

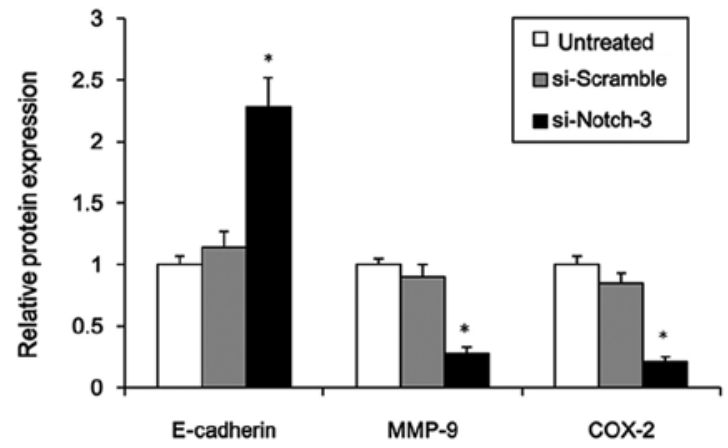

Figure 6. Notch1 knockdown regulates the expression of apoptosis-related genes and metastasis-associated molecules in hepatocellular carcinoma (HCC) cells. (A) Western blot analysis of Bcl-2 and BAX protein expression in $\mathrm{H} 22$ cells following Notch1 knockdown. (B) The relative protein expression of Bcl-2 and BAX in H22 cells following Notch1 knockdown. (C) Western blot analysis of E-cadherin, MMP-9 and COX-2 protein expression in H22 cells following Notch1 knockdown. (D) The relative protein expression of E-cadherin, MMP-9 and COX-2 in H22 cells following Notch1 knockdown. Data are presented as the means \pm SD. ${ }^{*}<0.05$.

cell viability and enhanced the apoptosis and metastatic capacity of HCC cells. We suggest that APS induced HCC cell apoptosis by suppressing the expression of Notch1.

The root of A. membranaceus, known as Huang Qi in Mandarin, was widely used in traditional Chinese medicine. APS, an extract of A. membranaceus, was extensively used in the clinic due to a number of beneficial effects including stimulation of the immune response, antiviral and antioxidant effects, protection against organ damage and inhibition of cancer cell proliferation (14-20,38). In the present study, we found that APS decreased H22 cell survival and induced apoptosis in a concentration-dependent manner (Fig. 2). Furthermore, the expression of apoptosis-related genes (Bcl-2 and BAX) and proteases (caspase- 3 and -8 ) was regulated by APS treatment (Fig. 3). APS decreased HCC cell survival through an apoptotic mechanism. A previous study reported that Astragalus saponin extract exerted anti-apoptotic effects on human peritoneal mesothelial cells during peritoneal gastric cancer metastasis and thus revealed that Astragalus may be used as an adjuvant chemotherapeutic agent in gastric cancer therapy (25). Astragalus saponin extract inhibited cell proliferation through cell cycle arrest, and promoted apoptosis in human colon cancer cells (HT-29) through caspase-3 activation and poly(ADP-ribose) polymerase cleavage (27). APS exerted a synergistic antitumor effect with adriamycin in H22 tumor-bearing mice through regulating cytokine and P-GP expression (28). Compound Astragalus and Salvia miltiorrhiza extract inhibited cell invasion by modulating transforming growth factor (TGF)- $\beta /$ Smad signaling in HepG2 cells (24). These findings suggest that APS has the potential to act as an effective chemotherapeutic agent in HCC treatment, and also to be used as an adjuvant in combination with other orthodox chemotherapeutic drugs in order to reduce side-effects.

In recent years, there have been several studies examining the biological activity of polysaccharides. However, to the best of our knowledge there have been limited reports regarding the regulatory mechanism underlying the antitumor effect of polysaccharides. Wang et al (36) demonstrated that S-GFB, a chemically sulfated polysaccharide obtained from Grifola frondosa, induced HepG2 cell proliferation and apoptosis through the Notch1/NF- $\mathrm{kB} / \mathrm{p} 65$-mediated caspase pathway. Previous research has indicated that Notch1 signaling modulated cell proliferation, apoptosis and differentiation in lung carcinoma and was associated with the progression of glioma $(33,34)$. Notably, it was found that Notch1 expression was significantly upregulated in the HCC tissues compared with that in the normal tissues (Fig. 1). Based on these findings, we speculated that APS may regulate the progression of HCC through Notch1 signaling. Our results confirmed this hypothesis. Firstly, APS treatment significantly decreased the mRNA and protein levels of Notch1 in the H22 cells in a concentration-dependent manner (Fig. 4), suggesting that APS induces apoptosis through decreasing Notch1 expression. In addition, Notch1 knockdown decreased cell viability and significantly increased the apoptosis of $\mathrm{H} 22$ cells by decreasing the expression of the apoptosis suppressor gene Bcl-2 and increasing the expression of the pro-apoptotic gene BAX (Figs. 5 and 6). Additonally, the protein level of E-cadherin was significantly upregulated by Notch1 knockdown, whereas the levels of MMP-9 and COX-2 were markedly reduced (Fig. 6C and D). It has been reported 
that in some types of cancer, COX-2 regulates the expression of E-cadherin (29). E-cadherin and MMP-9 are common metastasis-associated molecules. Our results demonstrated that Notch1 regulated the survival and metastatic capacity of HCC cells. APS induced the apoptosis of HCC cells by suppressing the expression of Notch1. Notch1 may be a potential therapeutic target for the treatment of HCC.

\section{References}

1. Jemal A, Bray F, Center MM, Ferlay J, Ward E and Forman D: Global cancer statistics. CA Cancer J Clin 61: 69-90, 2011.

2. El-Serag HB and Mason AC: Rising incidence of hepatocellular carcinoma in the United States. N Engl J Med 340: 745-750, 1999.

3. Parkin DM, Bray F, Ferlay J and Pisani P: Estimating the world cancer burden: Globocan 2000. Int J Cancer 94: 153-156, 2001.

4. Farazi PA and DePinho RA: Hepatocellular carcinoma pathogenesis: from genes to environment. Nat Rev Cancer 6: 674-687, 2006.

5. Fares $\mathrm{N}$ and Peron JM: Epidemiology, natural history, and risk factors of hepatocellular carcinoma. Rev Prat 63: 216-217, 220-222, 2013 (In French).

6. Cusnir M and Patt YZ: Novel systemic therapy options for hepatocellular carcinoma. Cancer J 10: 97-103, 2004

7. Takayasu K, Arii S, Ikai I, Omata M, Okita K, Ichida T, Matsuyama Y, Nakanuma Y, Kojiro M, Makuuchi M and Yamaoka Y; Liver Cancer Study Group of Japan: Prospective cohort study of transarterial chemoembolization for unresectable hepatocellular carcinoma in 8510 patients. Gastroenterology 131: 461-469, 2006

8. Han SH, Reddy KR, Keeffe EB, Soldevila-Pico C, Gish R, Chung RT, Degertekin B and Lok A; NIH HBV OLT Study Group: Clinical outcomes of liver transplantation for HBV-related hepatocellular carcinoma: data from the NIH HBV OLT study. Clin Transplant 25: E152-E162, 2011.

9. Rahbari NN, Mehrabi A, Mollberg NM, Müller SA, Koch M, Büchler MW and Weitz J: Hepatocellular carcinoma: current management and perspectives for the future. Ann Surg 253: 453-469, 2011.

10. Hassan T, El-Shafie M, Helmy A, Ammar S and Attyia M Liver resection for early stage hepatocellular carcinoma. SEC Oncology, 2015. DOI: 10.18056/seci2015.1.

11. Forner A, Hessheimer AJ, Isabel Real M and Bruix J: Treatment of hepatocellular carcinoma. Crit Rev Oncol Hematol 60: 89-98, 2006.

12. Silva MF and Wigg AJ: Current controversies surrounding liver transplantation for hepatocellular carcinoma. J Gastroenterol Hepatol 25: 1217-1226, 2010.

13. Sapisochin G, Castells L, Dopazo C, Bilbao I, Minguez B Lázaro JL, Allende H, Balsells J, Caralt M and Charco R: Single HCC in cirrhotic patients: liver resection or liver transplantation? Long-term outcome according to an intention-to-treat basis. Ann Surg Oncol 20: 1194-1202, 2013.

14. Guo L, Liu J, Hu Y, Wang D, Li Z, Zhang J, Qin T, Liu X, Liu C, Zhao X, et al: Astragalus polysaccharide and sulfated epimedium polysaccharide synergistically resist the immunosuppression. Carbohydr Polym 90: 1055-1060, 2012

15. Li HQ, Reeve-Johnson L and Wang JD: Effect of Astragalus polysaccharides on erythrocyte immune adherence of chickens inoculated with infectious bursal disease virus. Agric Sci China 6: 1402-1408, 2007.

16. Li R, Chen WC, Wang WP, Tian WY and Zhang XG: Antioxidant activity of Astragalus polysaccharides and antitumour activity of the polysaccharides and siRNA. Carbohydr Polym 82: 240-244, 2010.

17. Jin M, Zhao K, Huang Q and Shang P: Structural features and biological activities of the polysaccharides from Astragalus membranaceus. Int J Biol Macromol 64: 257-266, 2014.

18. Wang T, Sun Y, Jin L, Xu Y, Wang L, Ren T and Wang K: Enhancement of non-specific immune response in sea cucumber (Apostichopus japonicus) by Astragalus membranaceus and its polysaccharides. Fish Shellfish Immunol 27: 757-762, 2009.
19. Yin X, Chen L, Liu Y, Yang J, Ma C, Yao Z, Yang L, Wei L and $\mathrm{Li}$ M: Enhancement of the innate immune response of bladder epithelial cells by Astragalus polysaccharides through upregulation of TLR4 expression. Biochem Biophys Res Commun 397: 232-238, 2010.

20. Zhong R, Yu M, Liu H, Sun H, Cao Y and Zhou D: Effects of dietary Astragalus polysaccharide and Astragalus membranaceus root supplementation on growth performance, rumen fermentation, immune responses, and antioxidant status of lambs. Anim Feed Sci Technol 174: 60-67, 2012.

21. Chen Y, Song M, Wang Y, Xiong W, Zeng L, Zhang S, Xu M, Du H, Liu J, Wang D, et al: The anti-DHAV activities of Astragalus polysaccharide and its sulfate compared with those of BSRPS and its sulfate. Carbohydr Polym 117: 339-345, 2015.

22. Huang X, Wang D, Hu Y, Lu Y, Guo Z, Kong X and Sun J: Effect of sulfated astragalus polysaccharide on cellular infectivity of infectious bursal disease virus. Int J Biol Macromol 42: 166-171, 2008.

23. Li Q, Bao JM, Li XL, Zhang T and Shen XH: Inhibiting effect of Astragalus polysaccharides on the functions of $\mathrm{CD} 4{ }^{+} \mathrm{CD} 25$ highTreg cells in the tumor microenvironment of human hepatocellular carcinoma. Chin Med J (Engl) 125: 786-793, 2012.

24. Liu X, Yang Y, Zhang X, Xu S, He S, Huang W and Roberts MS: Compound Astragalus and Salvia miltiorrhiza extract inhibits cell invasion by modulating transforming growth factor- $\beta / \mathrm{Smad}$ in HepG2 cell. J Gastroenterol Hepatol 25: 420-426, 2010.

25. Na D, Liu FN, Miao ZF, Du ZM and Xu HM: Astragalus extract inhibits destruction of gastric cancer cells to mesothelial cells by anti-apoptosis. World J Gastroenterol 15: 570-577, 2009.

26. Zhu ZY, Liu RQ, Si CL, Zhou F, Wang YX, Ding LN, Jing C, Liu $\mathrm{AJ}$ and Zhang YM: Structural analysis and anti-tumor activity comparison of polysaccharides from Astragalus. Carbohydr Polym 85: 895-902, 2011

27. Tin MM, Cho CH, Chan K, James AE and Ko JK: Astragalus saponins induce growth inhibition and apoptosis in human colon cancer cells and tumor xenograft. Carcinogenesis 28: 1347-1355, 2007.

28. Tian QE, Li HD, Yan M, Cai HL, Tan QY and Zhang WY: Astragalus polysaccharides can regulate cytokine and P-glycoprotein expression in $\mathrm{H} 22$ tumor-bearing mice. World J Gastroenterol 18: 7079-7086, 2012.

29. Zhou L, Zhang N, Song W, You N, Li Q, Sun W, Zhang Y, Wang D and Dou K: The significance of Notch1 compared with Notch 3 in high metastasis and poor overall survival in hepatocellular carcinoma. PLoS One 8: e57382, 2013.

30. Zhu MS, Xu LB, Zeng H, Shi XD, Wu WR and Liu C: Association of Notch1 with vasculogenic mimicry in human hepatocellular carcinoma cell lines. Int J Clin Exp Pathol 7: 5782-5791, 2014.

31. Yoshida R, Nagata M, Nakayama H, Niimori-Kita K, Hassan W, Tanaka T, Shinohara M and Ito T: The pathological significance of Notch1 in oral squamous cell carcinoma. Lab Invest 93: 1068-1081, 2013

32. Roy M, Pear WS and Aster JC: The multifaceted role of Notch in cancer. Curr Opin Genet Dev 17: 52-59, 2007.

33. Jiang L, Wu J, Chen Q, Hu X, Li W and Hu G: Notch1 expression is upregulated in glioma and is associated with tumor progression. J Clin Neurosci 18: 387-390, 2011.

34. Wael H, Yoshida R, Kudoh S, Hasegawa K, Niimori-Kita K and Ito T: Notch1 signaling controls cell proliferation, apoptosis and differentiation in lung carcinoma. Lung Cancer 85: 131-140, 2014.

35. Wang M, Wu L, Wang L and Xin X: Down-regulation of Notch1 by gamma-secretase inhibition contributes to cell growth inhibition and apoptosis in ovarian cancer cells A2780. Biochem Biophys Res Commun 393: 144-149, 2010.

36. Wang CL, Meng M, Liu SB, Wang LR, Hou LH and Cao XH: A chemically sulfated polysaccharide from Grifola frondosa induces HepG2 cell apoptosis by notch1-NF- $\mathrm{kB}$ pathway. Carbohydr Polym 95: 282-287, 2013

37. Zhang JX, Han YP, Bai C and Li Q: Notch1/3 and p53/p21 are a potential therapeutic target for APS-induced apoptosis in non-small cell lung carcinoma cell lines. Int J Clin Exp Med 8: 12539-12547, eCollection 2015.

38. Yuan $\mathrm{CH}$, Liu YF and Cheng Y: Experimental study on effect of Astragalus extractum on canine isolated kidney during hypothermia perfusion and preservation. Zhongguo Zhong Xi Yi Jie He Za Zhi 23: 291-293, 2003 (In Chinese). 